\title{
Characterization and Lower Bounds for Branching Program Size Using Projective Dimension
}

\author{
Krishnamoorthy Dinesh ${ }^{1}$, Sajin Koroth ${ }^{2}$, and Jayalal Sarma ${ }^{3}$ \\ 1 Indian Institute of Technology Madras, Chennai, India \\ 2 Indian Institute of Technology Madras, Chennai, India \\ 3 Indian Institute of Technology Madras, Chennai, India
}

\begin{abstract}
We study projective dimension, a graph parameter (denoted by $\operatorname{pd}(G)$ for a graph $G$ ), introduced by Pudlák and Rödl (1992). For a Boolean function $f$ (on $n$ bits), Pudlák and Rödl associated a bipartite graph $G_{f}$ and showed that size of the optimal branching program computing $f$ (denoted by bpsize $(f)$ ) is at least $\operatorname{pd}\left(G_{f}\right)$ (also denoted by $\operatorname{pd}(f)$ ). Hence, proving lower bounds for $\operatorname{pd}(f)$ imply lower bounds for bpsize $(f)$. Despite several attempts (Pudlák and Rödl (1992), Rónyai et.al, (2000)), proving super-linear lower bounds for projective dimension of explicit families of graphs has remained elusive.

We observe that there exist a Boolean function $f$ for which the gap between the $\operatorname{pd}(f)$ and bpsize $(f))$ is $2^{\Omega(n)}$. Motivated by the argument in Pudlák and Rödl (1992), we define two variants of projective dimension - projective dimension with intersection dimension 1 (denoted by upd $(f)$ ) and bitwise decomposable projective dimension (denoted by $\operatorname{bpdim}(f)$ ). We show the following results:
\end{abstract}

(a) We observe that there exist a Boolean function $f$ for which the gap between upd $(f)$ and bpsize $(f)$ is $2^{\Omega(n)}$. In contrast, we also show that the bitwise decomposable projective dimension characterizes size of the branching program up to a polynomial factor. That is, there exists a large constant $c>0$ and for any function $f$,

$$
\operatorname{bpdim}(f) / 6 \leq \operatorname{bpsize}(f) \leq(\operatorname{bpdim}(f))^{c} .
$$

(b) We introduce a new candidate function family $f$ for showing super-polynomial lower bounds for $\operatorname{bpdim}(f)$. As our main result, we demonstrate gaps between $\operatorname{pd}(f)$ and the above two new measures for $f$ :

$$
\operatorname{pd}(f)=O(\sqrt{n}) \quad \operatorname{upd}(f)=\Omega(n) \quad \operatorname{bpdim}(f)=\Omega\left(\frac{n^{1.5}}{\log n}\right) .
$$

(c) Although not related to branching program lower bounds, we derive exponential lower bounds for two restricted variants of $\mathrm{pd}(f)$ and $\mathrm{upd}(f)$ respectively by observing that they are exactly equal to well-studied graph parameters - bipartite clique cover number and bipartite partition number respectively.

1998 ACM Subject Classification F.1.1 Models of Computation, F.1.3 Complexity Measures and Classes

Keywords and phrases Projective Dimension, Lower Bounds, Branching Program Size.

Digital Object Identifier 10.4230/LIPIcs.FSTTCS.2016.37

\section{Introduction}

A central question in complexity theory - the $\mathrm{P}$ vs $\mathrm{L}$ problem - asks if a deterministic Turing machine that runs in polynomial time can accept any language that cannot be accepted

c) (i) Krishnamoorthy Dinesh, Sajin Koroth, and Jayalal Sarma;

c. licensed under Creative Commons License CC-BY

36th IARCS Annual Conference on Foundations of Software Technology and Theoretical Computer Science (FSTTCS 2016).

Editors: Akash Lal, S. Akshay, Saket Saurabh, and Sandeep Sen; Article No. 37; pp. 37:1-37:14

Leibniz International Proceedings in Informatics

LIPICS Schloss Dagstuhl - Leibniz-Zentrum für Informatik, Dagstuhl Publishing, Germany 
by deterministic Turing machines with logarithmic space bound. A stronger version of the problem asks if $\mathrm{P}$ is separate from $\mathrm{L} /$ poly (deterministic logarithmic space given polynomial sized advice). The latter, recast in the language of circuit complexity theory, asks if there exists an explicit family of functions $\left(f:\{0,1\}^{n} \rightarrow\{0,1\}\right)$ computable in polynomial time (in terms of $n$ ), such that any family of deterministic branching programs computing them has to be of size $2^{\Omega(n)}$. However, the best known non-trivial size lower bound against deterministic branching programs, due to Nechiporuk [11] in 1970s, is $\Omega\left(\frac{n^{2}}{\log ^{2} n}\right)$.

Pudlák and Rödl [12] described a linear algebraic approach to show size lower bounds against deterministic branching programs. They introduced a linear algebraic parameter called projective dimension (denoted by $\operatorname{pd}_{\mathbb{F}}(f)$, over a field $\mathbb{F}$ ) defined on a natural graph associated with the Boolean function $f$. For a Boolean function $f:\{0,1\}^{2 n} \rightarrow\{0,1\}$, fix a partition of the input bits into two parts of size $n$ each, and consider the bipartite graph $G_{f}(U, V, E)$ defined on vertex sets $U=\{0,1\}^{n}$ and $V=\{0,1\}^{n}$, as $(u, v) \in E$ if and only if $f(u v)=1$. We call $G_{f}$ as the bipartite realization of $f$. For a bipartite graph $G(U, V, E)$, the projective dimension of $G$ over a field $\mathbb{F}$, denoted by $\operatorname{pd}_{\mathbb{F}}(G)$, is defined as the smallest $d$ for which there is a vector space $W$ of dimension $d$ (over $\mathbb{F}$ ) and a function $\phi$ mapping vertices in $U, V$ to linear subspaces of $W$ such that for all $(u, v) \in U \times V,(u, v) \in E$ if and only if $\phi(u) \cap \phi(v) \neq\{0\}$. We say that $\phi$ realizes the graph $G$.

Pudlák and Rödl [12] showed that if $f$ can be computed by a deterministic branching program of size $s$, then $\operatorname{pd}_{\mathbb{F}}(f) \leq s$ over any field $\mathbb{F}$. Thus, in order to establish size lower bounds against branching programs, it suffices to prove lower bounds for projective dimension of explicit family of Boolean functions.

By a counting argument, Pudlák and Rödl in [12] showed that for most Boolean functions $f:\{0,1\}^{n} \times\{0,1\}^{n} \rightarrow\{0,1\}, \operatorname{pd}_{\mathbb{R}}(f)$ is $\Omega\left(\sqrt{\frac{2^{n}}{n}}\right)$. In a subsequent work, the same authors [13] also established an upper bound $\operatorname{pd}_{\mathbb{R}}(f)=O\left(\frac{2^{n}}{n}\right)$ for all functions. More recently, Rónyai, Babai and Ganapathy [15] established the same lower bound over all fields. Over finite fields $\mathbb{F}$, Pudlák and Rödl [12] also showed (by a counting argument) that there exists a Boolean function $f:\{0,1\}^{n} \times\{0,1\}^{n} \rightarrow\{0,1\}$ such that $\operatorname{pd}_{\mathbb{F}}(f)$ is $\Omega\left(\sqrt{2^{n}}\right)$. However, till date, obtaining an explicit family of Boolean functions (equivalently graphs) achieving such lower bounds remain elusive. The best lower bound for projective dimension for an explicit family of functions is for the inequality function (on $2 n$ bits, the graph is the bipartite complement of the perfect matching) where a lower bound of $\epsilon n$ for an absolute constant $\epsilon>0$ is known [12] over $\mathbb{R}$. For a survey on projective dimension and related linear algebraic techniques, refer $[13,9]$. However, the best known size lower bound that was achieved using this framework is only $\Omega(n)$ which is not better than trivial lower bounds.

Our Results: Our starting point is the observation that projective assignment appearing in the proof of [12] also has the property that the dimension of the intersection of two subspaces assigned to the vertices is exactly 1, whenever they intersect (See Proposition 2.2(2)). We denote, for a function $f$, the variant of projective dimension defined by this property as $\operatorname{upd}(f)$ (see Section 4). From the above discussion, for any Boolean function $f, \operatorname{pd}(f) \leq$ $\operatorname{upd}(f) \leq \operatorname{bpsize}(f)$. A natural question is whether this restriction helps in proving better lower bounds for the branching programs. By observing properties about the measure of projective dimension, choosing a new candidate function ${ }^{1}$, we demonstrate that the above restriction can help by proving the following quadratic gap between the two measures.

1 The candidate function is in $N C^{2}$ but unlikely to be in NL. 
- Theorem 1.1. For any $d \geq 0$, for the function $\mathrm{SI}_{d}$ (on $2 d^{2}$ variables, see Definition 2.3), the projective dimension is exactly equal to $d$, while the projective dimension with intersection dimension 1 is $\Omega\left(d^{2}\right)$.

However, this does not directly improve the known branching program size lower bound for $\mathrm{SI}_{d}$, since it leads to only a linear lower bound on upd $\left(\mathrm{SI}_{d}\right)$. We demonstrate the weakness of this measure by showing the existence of a function (although not explicit) for which there is an exponential gap between upd over any partition and the branching program size (Proposition 5.1). This motivates us to look for variants of projective dimension of graphs, which is closer to the optimal branching program size of the corresponding Boolean function. We observe more properties (see Proposition 2.2) about the subspace assignment from the proof of the upper bound from [12]. We call the projective assignments with these properties bitwise decomposable projective assignment and denote the corresponding dimension ${ }^{2}$ as $\operatorname{bitpdim}(f)$ (See Definition 5.2). Thus, for any Boolean function $f, \operatorname{pd}(f) \leq \operatorname{bitpdim}(f)$. We also show that $\operatorname{bitpdim}(f) \leq 6 \cdot \operatorname{bpsize}(f)$ (Lemma 5.3). To demonstrate the tightness of the definition, we first argue a converse with respect to this new parameter.

- Theorem 1.2. There is an absolute constant $c>0$ such that if $\operatorname{bitpdim}\left(f_{n}\right) \leq d(n)$ for a function family $\left\{f_{n}\right\}_{n \geq 0}$ on $2 n$ bits, then there is a deterministic branching program of size $(d(n))^{c}$ computing it.

Thus, super-polynomial size lower bounds for branching programs imply super-polynomial lower bounds for bitpdim $(f)$. The function $\mathrm{SI}_{d}$ (on $2 d^{2}$ input bits - see Definition 2.3) is a natural candidate for proving bitpdim lower bounds as the corresponding language is hard ${ }^{3}$ for the complexity class $C_{=} L$ under logspace Turing reductions.

However, the best known lower bound for branching program size for an explicit family of functions is $\Omega\left(\frac{n^{2}}{\log ^{2} n}\right)$ by Nechiporuk [11] which uses a counting argument on the number of sub-functions. By Theorem 1.2, $\operatorname{bitpdim}(f)$ (for the same explicit function) is at least $\Omega\left(\frac{n^{2 / c}}{\log ^{2 / c} n}\right)$. The constant $c$ is large $e^{4}$ and hence implies only weak lower bounds for bitpdim. Despite this weak connection, by combining the counting strategy with the linear algebraic structure of bitpdim, we show a super-linear lower bound for $\mathrm{SI}_{d}$ matching the branching program size lower bound 5 .

- Theorem 1.3 (Main Result). For any $d>0$, bitpdim( $\left(\mathrm{SI}_{d}\right)$ is at least $\Omega\left(\frac{d^{3}}{\log d}\right)$.

Theorems 1.1 and 1.3 demonstrate gaps between the pd and the new measures considered. In particular, for $n=d^{2}, \operatorname{pd}\left(\mathrm{SI}_{d}\right)=O(\sqrt{n}), \operatorname{upd}\left(\mathrm{SI}_{d}\right)=\Omega(n)$, and $\operatorname{bitpdim}\left(\mathrm{SI}_{d}\right)=\Omega\left(\frac{n^{1.5}}{\log n}\right)$. We remark that Theorem 1.3 implies a size lower bound of $\Omega\left(\frac{n^{1.5}}{\log n}\right)$ for branching programs computing the function $\mathrm{SI}_{d}$ (where $n=d^{2}$ ). However, note that this can also be derived from Nechiporuk's method. For the Element Distinctness function, the above linear algebraic adaptation of Nechiporuk's method for bitpdim gives $\Omega\left(\frac{n^{2}}{\log ^{2} n}\right)$ lower bounds (for bitpdim and hence for bpsize) which matches with the best lower bound that Nechiporuk's method can derive. This shows that our modification of approach in [12] can also achieve the best known lower bounds for branching program size.

2 We do not use the property that intersection dimension is 1 and hence is incomparable with upd.

3 Assuming $\mathrm{C}=\mathrm{L} \nsubseteq \mathbb{L} /$ poly, $\mathrm{SI}_{d}$ cannot be computed by deterministic branching programs of size poly $(d)$.

${ }^{4}$ However, the value of $c$ can be shown to be at most 5. See proof of Theorem 1.2 in Section 5.1.

5 A lower bound of $\Omega\left(\frac{d^{3}}{\log d}\right)$ for the branching program size can also be obtained using Nechiporuk's method. 
Continuing the quest for better lower bounds for projective dimension, we study two further restrictions. In these variants of pd and upd, the subspaces assigned to the vertices must be spanned by standard basis vectors. We denote the corresponding dimensions as $\operatorname{spd}(f)$ and $\operatorname{uspd}(f)$ respectively. It is easy to see that for any $2 n$-bit function, both of these dimensions are upper bounded by $2^{n}$.

We connect these variants to some of the well-studied graph parameters. The bipartite clique cover number (denoted by $b c(G)$ ) is the smallest collection of complete bipartite subgraphs of $G$ such that every edge in $G$ is present in some graph in the collection. If we insist that the bipartite graphs in the collection be edge-disjoint, the measure is called bipartite partition number denoted by $b p(G)$. By definition, $b c(G) \leq b p(G)$. These graph parameters are closely connected to communication complexity as well. More precisely, $\log \left(b c\left(G_{f}\right)\right)$ is exactly the non-deterministic communication complexity of the function $f$, and $\log \left(b p\left(G_{f}\right)\right)$ is a lower bound on the deterministic communication complexity of $f$ (see $[6])$. In this context, we show the following:

- Theorem 1.4. For any Boolean function $f, \operatorname{spd}(f)=b c\left(G_{f}\right)$ and $\operatorname{uspd}(f)=b p\left(G_{f}\right)$.

Thus, if for a function family, the non-deterministic communication complexity is $\Omega(n)$, then we will have $\operatorname{spd}(f)=2^{\Omega(n)}$. Thus, both spd(DISJ) and uspd(DISJ) are $2^{\Omega(n)}$.

\section{Preliminaries}

In this section, we introduce the notations used in the paper. For definitions of basic complexity classes and computational models, we refer the reader to standard textbooks $[6$, $16]$.

Unless otherwise stated we work over the field $\mathbb{F}_{2}$. We remark that our arguments do generalize to any finite field. All subspaces that we talk about in this work are linear subspaces. Also $\overrightarrow{0}$ and $\{0\}$ denote the zero vector, and zero-dimensional space respectively. For a subspace $U \subseteq \mathbb{F}^{n}$, we call the ambient dimension of $U$ as $n$. We denote $e_{i} \in \mathbb{F}^{n}$ as the $i^{\text {th }}$ standard basis vector with $i^{\text {th }}$ entry being 1 and rest of the entires being zero.

For a graph $G(U, V, E)$, recall the definition of projective dimension of $G$ over a field $\mathbb{F}\left(\operatorname{pd}_{\mathbb{F}}(G)\right)$, defined in the introduction. For a Boolean function $f:\{0,1\}^{2 n} \rightarrow\{0,1\}$, fix a partition of the input bits into two parts of size $n$ each, and consider the bipartite graph $G_{f}$ defined on vertex sets $U=\{0,1\}^{n}$ and $V=\{0,1\}^{n}$, as $(u, v) \in E$ if and only if $f(u v)=1$. A $\phi$ is said to realize the function $f$ if it realizes $G_{f}$. Unless otherwise mentioned, the partition is the one specified in the definition of the function. We denote by bpsize $(f)$ the number of vertices (including accept and reject nodes) in the optimal branching program computing $f$.

- Theorem 2.1 (Pudlák-Rödl Theorem [12]). For a Boolean function $f$ computed by a deterministic branching program of size $s$ and $\mathbb{F}$ being any field, $\operatorname{pd}_{\mathbb{F}}\left(G_{f}\right) \leq s$.

The proof of this result proceeds by producing a subspace assignment for vertices of $G_{f}$ from a branching program computing $f$. We derive the following proposition by a careful analysis of the aforementioned proof in [12].

- Proposition 2.2. For a Boolean function $f:\{0,1\}^{n} \times\{0,1\}^{n} \rightarrow\{0,1\}$ computed by a deterministic branching program of size $s$, there is a collection of subspaces of $\mathbb{F}^{s}$ denoted $\mathcal{C}=\left\{U_{i}^{a}\right\}_{i \in[n], a \in\{0,1\}}$ and $\mathcal{D}=\left\{V_{j}^{b}\right\}_{j \in[n], b \in\{0,1\}}$, where we associate the subspace $U_{i}^{a}$ with $a$ bit assignment $x_{i}=a$ and $V_{j}^{b}$ with $y_{j}=b$ such that if we define the map $\phi$ assigning subspaces from $\mathbb{F}^{s}$ to vertices of $G_{f}(U, V, E)$ as $\phi(x)=\operatorname{span}\left\{U_{i}^{x_{i}}\right\}, \phi(y)=\operatorname{span}\left\{V_{j}^{y_{j}}\right\}$, for $x \in X, y \in Y$ then the following holds true. Let $S=\left\{e_{i}-e_{j} \mid i, j \in[s], i \neq j\right\}$. 
1. for all $(u, v) \in U \times V, \phi(u) \cap \phi(v) \neq\{0\}$ if and only if $f(u, v)=1$.

2. for all $(u, v) \in U \times V$, $\operatorname{dim}(\phi(u) \cap \phi(v)) \leq 1$.

3. For any $W \in \mathcal{C} \cup \mathcal{D}, \exists S^{\prime} \subseteq S$ such that $W=\operatorname{span}\left\{S^{\prime}\right\}$.

We define the following family of functions and family of graphs based on subspaces of a vector space and their intersections.

- Definition $2.3\left(\mathrm{SI}_{d}, \mathcal{P}_{d}\right)$. Let $\mathbb{F}$ be a finite field. Denote by $\mathrm{SI}_{d}$, the Boolean function defined on $\mathbb{F}^{d \times d} \times \mathbb{F}^{d \times d} \rightarrow\{0,1\}$ as for any $A, B \in \mathbb{F}^{d \times d} \operatorname{SI}_{d}(A, B)=1$ if and only if $\operatorname{rowspan}(A) \cap \operatorname{rowspan}(B) \neq\{0\}$. Note that the row span is over the field $\mathbb{F}$ (which, in our case, is $\left.\mathbb{F}_{2}\right)$. Denote by $\mathcal{P}_{d}$, the bipartite graph $(U, V, E)$ where $U$ and $V$ are the set of all subspaces of $\mathbb{F}^{d}$. And for any $(I, J) \in U \times V,(I, J) \in E \Longleftrightarrow I \cap J \neq\{0\}$

We collect the definitions of Boolean functions which we deal with in this work. For $(x, y) \in\{0,1\}^{n} \times\{0,1\}^{n}, \mathrm{IP}_{n}(x, y)=\sum_{i=1}^{n} x_{i} y_{i} \bmod 2, \mathrm{EQ}_{n}(x, y)$ is 1 if $\forall i \in[n] x_{i}=y_{i}$ and is 0 otherwise, $\operatorname{INEQ}_{n}(x, y)=\neg \mathrm{EQ}_{n}(x, y)$ and $\operatorname{DISJ}_{n}(x, y)=1$ if $\forall i \in[n] x_{i} \wedge y_{i}=0$ and is 0 otherwise. Note that all the functions discussed so far has branching programs of size $O(n)$ computing them and hence have projective dimension $O(n)$ by Theorem 2.1 .

Let $m \in \mathbb{N}$ and $n=2 m \log m$. The Boolean function, Element Distinctness, denoted $\mathrm{ED}_{n}$ is defined on $2 m$ blocks of $2 \log m$ bits, $x_{1}, \ldots, x_{m}$ and $y_{1}, \ldots, y_{m}$ bits and it evaluates to 1 if and only if all the $x_{i}$ s and $y_{i}$ s take distinct values when interpreted as integers in $\left[\mathrm{m}^{2}\right]$. Let $q$ be a power of prime congruent to 1 modulo 4 . Identify elements in $\{0,1\}^{n}$ with elements of $\mathbb{F}_{q}^{*}$. For $x, y \in \mathbb{F}_{q}^{*}$, the Paley function $\operatorname{PAL}_{n}^{q}(x, y)=1$ if $x-y$ is a quadratic residue in $\mathbb{F}_{q}^{*}$ and 0 otherwise.

We observe for any induced subgraph $H$ of $G$, if $G$ is realized in a space of dimension $d$, then $H$ can also be realized in a space of dimension $d$. For any $d \in \mathbb{N}, \mathcal{P}_{d}$ appears as an induced subgraph of the bipartite realization of $\mathrm{SI}_{d}$. Hence, $\operatorname{pd}\left(\mathrm{SI}_{d}\right) \geq \operatorname{pd}\left(\mathcal{P}_{d}\right)$.

\section{Properties of Projective Dimension}

In this section, we observe properties about projective dimension as a measure of graphs and Boolean functions. We start by proving closure properties of projective dimension under Boolean operations $\wedge$ and $\vee$.

- Lemma 3.1. Let $\mathbb{F}$ be an arbitrary field. For any two functions $f_{1}:\{0,1\}^{2 n} \rightarrow\{0,1\}, f_{2}$ : $\{0,1\}^{2 n} \rightarrow\{0,1\}, \operatorname{pd}_{\mathbb{F}}\left(f_{1} \vee f_{2}\right) \leq \operatorname{pd}_{\mathbb{F}}\left(f_{1}\right)+\operatorname{pd}_{\mathbb{F}}\left(f_{2}\right)$ and $\operatorname{pd}_{\mathbb{F}}\left(f_{1} \wedge f_{2}\right) \leq \operatorname{pd}_{\mathbb{F}}\left(f_{1}\right) \cdot \operatorname{pd}_{\mathbb{F}}\left(f_{2}\right)$

The proof is based on direct sum and tensor product of vector spaces. The $\vee$ part of the above lemma was also observed (without proof) in [13]. We remark that the construction for $\vee$ is tight up to constant factors. Assume $n$ is a multiple of 4 . Consider the functions $f\left(x_{1}, \ldots, x_{\frac{n}{4}}, x_{\frac{n}{2}+1}, \ldots, x_{\frac{3 n}{4}}\right)$ and $g\left(x_{\frac{n}{4}+1}, \ldots, x_{\frac{n}{2}}, x_{\frac{3 n}{4}+1}, \ldots, x_{n}\right)$ each of which performs inequality check on the first $\frac{n}{4}$ and the second $\frac{n}{4}$ variables. It is easy to see that $f \vee g$ is the inequality function on $\frac{n}{2}$ variables $x_{1}, \ldots, x_{\frac{n}{2}}$ and the next $\frac{n}{2}$ variables $x_{\frac{n}{2}+1}, \ldots, x_{n}$. By the fact that they are computed by $n$ size branching programs and using Theorem 2.1 (Pudlák-Rödl theorem) we get that $\operatorname{pd}(f) \leq n$ and $\operatorname{pd}(g) \leq n$. Hence by Lemma 3.1, $\operatorname{pd}(f \vee g) \leq \operatorname{pd}(f)+\operatorname{pd}(g) \leq 2 n$. Lower bound on projective dimension of inequality function comes from $\left[12\right.$, Theorem 4], giving $\operatorname{pd}(f \vee g) \geq \epsilon \cdot \frac{n}{2}$ for an absolute constant $\epsilon$. This shows that $\operatorname{pd}(f \vee g)=\Theta(n)$. We also cannot expect a general relation connecting $\operatorname{pd}_{\mathbb{R}}(f)$ and $\operatorname{pd}_{\mathbb{R}}(\neg f)$ since it is known [12] that $\operatorname{pd}_{\mathbb{R}}\left(\operatorname{INEQ}_{n}\right)$ is $\Omega(n)$ while $\operatorname{pd}_{\mathbb{R}}\left(\mathrm{EQ}_{n}\right)=2$.

We now observe a characterization of bipartite graphs having projective dimension at most $d$ over $\mathbb{F}$. 
- Lemma 3.2 (Characterization). Let $G$ be a bipartite graph with no two vertices having same neighborhood, $\operatorname{pd}(G) \leq d$ if and only if $G$ is an induced subgraph of $\mathcal{P}_{d}$.

It follows that $\operatorname{pd}\left(\mathcal{P}_{d}\right) \leq d$. Observe that, in any projective assignment, the vertices with different neighborhoods should be assigned different subspaces. For $\operatorname{pd}\left(\mathcal{P}_{d}\right)$, all vertices on either partitions have distinct neighborhoods. The number of subspaces of a vector space of dimension $d-1$ is strictly smaller than the number of vertices in $\mathcal{P}_{d}$. Thus, we conclude the following theorem.

- Theorem 3.3. For any $d \in \mathbb{N}, \operatorname{pd}\left(\mathcal{P}_{d}\right)=\operatorname{pd}\left(\mathrm{SI}_{d}\right)=d$.

For an $N$ vertex graph $G$, the number of vertices of distinct neighborhood can at most be $N$. Thus, the observation that we used to show the lower bound for the graph $\operatorname{pd}\left(\mathcal{P}_{d}\right)$ cannot be used to obtain more than a $\sqrt{\log N}$ lower bound for $\mathrm{pd}(G)$. Also, for many functions, the number of vertices of distinct neighborhood can be smaller.

We observe that by incurring an additive factor of $2 \log N$, any graph $G$ on $N$ vertices can be transformed into a graph $G^{\prime}$ on $2 N$ vertices such that all the neighborhoods of vertices in one partition are all distinct. Let $f:\{0,1\}^{2 n} \rightarrow\{0,1\}$ be such that the neighborhoods of $G_{f}$ are not necessarily distinct. We consider a new function $f^{\prime}$ whose bipartite realization will have two copies of $G_{f}$ namely $G_{1}\left(A_{1}, B_{1}, E_{1}\right)$ and $G_{2}\left(A_{2}, B_{2}, E_{2}\right)$ where $A_{1}, A_{2}, B_{1}, B_{2}$ are disjoint and a matching connecting vertices in $A_{1}$ to $B_{2}$ and $A_{2}$ to $B_{1}$ respectively. Since the matching edges associated with every vertex is unique, the neighborhoods of all vertices are bound to be distinct. Applying Lemma 3.1 and observing that matching (i.e, equality function) has projective dimension at most $n, \operatorname{pd}\left(f^{\prime}\right) \leq 2 \operatorname{pd}(f)+2 n$. This shows that to show super-linear lower bounds on projective dimension for $f$ where the neighborhoods may not be distinct, it suffices to show a super-linear lower bound for $f^{\prime}$.

\section{Projective Dimension with Intersection Dimension 1}

Motivated by the proof of Theorem 2.1, we make the following definition.

- Definition 4.1 (Projective Dimension with Intersection Dimension 1). A Boolean function $f:\{0,1\}^{n} \times\{0,1\}^{n} \rightarrow\{0,1\}$ with the corresponding bipartite graph $G(U, V, E)$ is said to have projective dimension with intersection dimension 1 (denoted by upd $(f)) d$ over field $\mathbb{F}$, if $d$ is the smallest possible dimension for which there exists a vector space $K$ of dimension $d$ over $\mathbb{F}$ with a map $\phi$ assigning subspaces of $K$ to $U \cup V$ such that

- for all $(u, v) \in U \times V, \phi(u) \cap \phi(v) \neq\{0\}$ if and only if $(u, v) \in E$.

- for all $(u, v) \in U \times V, \operatorname{dim}(\phi(u) \cap \phi(v)) \leq 1$.

By the properties observed in Proposition 2.2,

- Theorem 4.2. For a Boolean function $f$ computed by a deterministic branching program of size $s, \operatorname{upd}_{\mathbb{F}}(f) \leq s$ for any field $\mathbb{F}$.

Thus, it suffices to prove lower bounds for upd $(f)$ in order to obtain branching program size lower bounds. We now proceed to show lower bounds on upd. Our approaches use the fact that the adjacency matrix of $\mathcal{P}_{d}$ has high rank.

- Lemma 4.3. Let $M$ be the bipartite adjacency matrix of $\mathcal{P}_{d}$, then rank $(M) \geq\left[\begin{array}{c}d \\ d / 2\end{array}\right]_{q} \geq q^{\frac{d^{2}}{4}}$

Proof. For $0 \leq i \leq k \leq d$, and subspace $I, K \subseteq \subseteq_{s} \mathbb{F}_{q}^{d}$ with $\operatorname{dim}(I)=i, \operatorname{dim}(K)=k$, define matrix $\overline{W_{i k}}$ over $\mathbb{R}$ as $\overline{W_{i k}}(I, K)=1$ if $I \cap K=\{0\}$ and 0 otherwise. This matrix has dimension $\left[\begin{array}{l}d \\ i\end{array}\right]_{q} \times\left[\begin{array}{l}d \\ k\end{array}\right]_{q}$. 
Consider the submatrix $M_{i}$ of $M$ with rows and columns indexed by subspaces of dimension exactly $i$. Observe that $\overline{W_{i i}}=J-M_{i}$ where $J$ is an all ones matrix of appropriate order. These matrices are well-studied (see [5]). Closed form expressions for eigenvalues are computed in $[3,10]$ and the eigenvalues are known to be non-zero. Hence for $0 \leq i \leq d / 2$ the matrix $\overline{W_{i i}}$ has rank $\left[\begin{array}{l}d \\ i\end{array}\right]_{q}$. Since $\overline{W_{i i}}=J-M_{i}$, rank $\left(M_{i}\right) \geq \operatorname{rank}\left(\overline{W_{i i}}\right)-1$. This shows that $\operatorname{rank}(M) \geq \operatorname{rank}\left(M_{i}\right)=\left[\begin{array}{l}d \\ i\end{array}\right]_{q}$ for all $i$ such that $2 i \leq d$. Choosing $i=d / 2$ gives $\operatorname{rank}(M) \geq\left[\begin{array}{c}d \\ d / 2\end{array}\right]_{q}-1 \geq q^{\frac{d^{2}}{4}}-1$.

We now present two approaches for showing lower bounds on upd $(f)$ - one using intersection families of vector spaces and the other using rectangle arguments on $M_{f}$.

Lower Bound for upd $\left(\mathcal{P}_{\boldsymbol{d}}\right)$ using intersecting families of vector spaces: To prove a lower bound on $\operatorname{upd}\left(\mathcal{P}_{d}\right)$ we define a matrix $N$ from a projective assignment with intersection dimension 1 for $\mathcal{P}_{d}$, such that it is equal to $(q-1) M$. Let $D=\operatorname{upd}\left(\mathcal{P}_{d}\right)$. We first show that $\operatorname{rank}(N)$ is at most $1+\left[\begin{array}{c}D \\ 1\end{array}\right]_{q}$. Then by Lemma 4.3 we get that $\operatorname{rank}(N)$ is at least $q^{\frac{d^{2}}{4}}$. Let $\mathcal{G}=\left\{G_{1}, \ldots, G_{m}\right\}, \mathcal{H}=\left\{H_{1}, \ldots, H_{m}\right\}$ be the subspace assignment with intersection dimension 1 realizing $\mathcal{P}_{d}$ with dimension $D$.

- Lemma 4.4. For any polynomial $p$ in $q^{x}$ of degree $s$, with matrix $N$ of order $|\mathcal{G}| \times|\mathcal{H}|$ defined as $N\left[G_{r}, H_{t}\right]=p\left(\operatorname{dim}\left(G_{r} \cap H_{t}\right)\right)$ with $G_{r} \in \mathcal{G}, H_{t} \in \mathcal{H}$, then $\operatorname{rank}(N) \leq \sum_{i=0}^{s}\left[\begin{array}{c}D \\ i\end{array}\right]_{q}$

Proof. This proof is inspired by the proof in [4] of a similar claim where a non-bipartite version of this lemma is proved. To begin with, note that $p$ is a degree $s$ polynomial in $q^{x}$, and hence can be written as a linear combination of polynomials $p_{i}=\left[\begin{array}{c}x \\ i\end{array}\right]_{q}, 0 \leq i \leq s$. Let the linear combination be given by $p(x)=\sum_{i=0}^{s} \alpha_{i} p_{i}(x)$. For $0 \leq i \leq s$ define a matrix $N_{i}$ with rows and columns indexed respectively by $\mathcal{G}, \mathcal{H}$ defined as $N_{i}\left[G_{r}, H_{s}\right]=p_{i}\left(\operatorname{dim} G_{r} \cap H_{s}\right)$. By definition of $N_{i}, N=\sum_{i \in[s]} \alpha_{i} N_{i}$.

To bound the rank of $N_{i}$ 's we introduce the following families of inclusion matrices. For any $j \in[D]$, consider two matrices $\Gamma_{j}$ corresponding to $\mathcal{G}$ and $\Delta_{j}$ corresponding to $\mathcal{H}$ defined as $\Gamma_{j}(G, I)=1$ if $\operatorname{dim}(I)=j, G \in \mathcal{G}, I \subseteq_{s} G$ and 0 otherwise. $\Delta_{j}(H, I)=1$ if $\operatorname{dim}(I)=j, H \in \mathcal{H}, I \subseteq \subseteq_{s} H$ and 0 otherwise. Note that rank of the these matrices are upper bounded by the number of columns which is $\left[\begin{array}{c}D \\ j\end{array}\right]_{q}$. We claim that for any $i \in\{0,1, \ldots, s\}$, $\operatorname{rank}\left(N_{i}\right) \leq\left[\begin{array}{c}D \\ i\end{array}\right]_{q}$. This completes the proof since $N=\sum_{i \in[s]} \alpha_{i} N_{i}$.

To prove the claim, let $\mathcal{F}_{i}$ denote the set of all $i$ dimensional subspace of $\mathbb{F}_{q}^{D}$. We show that $N_{i}=\Gamma_{i} \Delta_{i}^{T}$. Hence rank $\left(N_{i}\right) \leq \min \left\{\operatorname{rank}\left(\Gamma_{i}\right), \operatorname{rank}\left(\Delta_{i}\right)\right\} \leq\left[\begin{array}{c}D \\ i\end{array}\right]_{q}$. For $\left(G_{r}, H_{t}\right) \in \mathcal{G} \times \mathcal{H}$, $\Gamma_{i} \Delta_{i}^{T}\left(G_{r}, H_{t}\right)=\sum_{I \in \mathcal{F}_{i}} \Gamma_{i}\left(G_{r}, I\right) \Delta_{i}^{T}\left(I, H_{t}\right)=\sum_{I \in \mathcal{F}_{i}} \Gamma_{i}\left(G_{r}, I\right) \Delta_{i}\left(H_{t}, I\right)=\sum_{I \in \mathcal{F}_{i}}\left[I \subseteq_{s}\right.$ $\left.G_{r}\right] \wedge\left[I \subseteq_{s} H_{t}\right]=\sum_{I \in \mathcal{F}_{i}}\left[I \subseteq_{s} G_{r} \cap H_{t}\right]=\left[\begin{array}{c}\operatorname{dim}\left(G_{r} \cap H_{t}\right) \\ i\end{array}\right]_{q}=N_{i}\left(G_{r}, H_{t}\right)$

We apply Lemma 4.4 on $N$ defined via $p(x)=q^{x}-1$ with $s=1$, to get $q^{d^{2} / 4} \leq\left[\begin{array}{c}d \\ d / 2\end{array}\right]_{q} \leq$ $1+\left[\begin{array}{c}D \\ 1\end{array}\right]_{q}=1+\left(q^{D}-1\right) /(q-1)$. By definition, $\operatorname{rank}(N)=\operatorname{rank}(M)$. This gives that $D=\Omega\left(d^{2}\right)$ and proves Theorem 1.1 .

Lower Bound for upd $\left(\mathcal{P}_{\boldsymbol{d}}\right)$ from Rectangle Arguments: We now give an alternate proof of for Theorem 1.1 using combinatorial rectangle arguments.

- Lemma 4.5. For $f:\{0,1\}^{n} \times\{0,1\}^{n} \rightarrow\{0,1\}$ with $M_{f}$ denoting the bipartite adjacency matrix of $G_{f}, \operatorname{rank}_{\mathbb{R}}\left(M_{f}\right) \leq q^{O\left(\operatorname{upd}_{\mathbb{F}}(f)\right)}$ where $\mathbb{F}$ is a finite field of size $q$. 
Proof. Let $\phi$ be a subspace assignment realizing $f$ of dimension $d$ with intersection dimension 1. Let $S(v)$ for $v \in \mathbb{F}_{q}^{d}$ denote $\left\{(a, b) \in\{0,1\}^{n} \times\{0,1\}^{n} \mid \phi(a) \cap \phi(b)=\operatorname{span}\{v\}\right\}$. Also let $M_{v}$ denote the matrix representation of $S(v)$. That is, $M_{v}(a, b)=1 \Longleftrightarrow(a, b) \in S(v)$. Consider all 1 dimensional subspaces which appear as intersection space for some input $(x, y)$. Fix a basis vector for each space and let $T$ denote the collection of basis vectors of all the intersection spaces. Note that for any $(x, y) \in f^{-1}(1)$, there is a unique $v \in \mathbb{F}_{q}^{d}$ (up to scalar multiples) such that $(x, y) \in S(v)$ for otherwise intersection dimension exceeds 1. Then $M_{f}=\sum_{v \in T} M_{v}$. Now, $\operatorname{rank}\left(M_{f}\right) \leq \sum_{v \in T} \operatorname{rank}\left(M_{v}\right)$. Since $\operatorname{rank}\left(M_{v}\right)=1$, $\operatorname{rank}\left(M_{f}\right) \leq|T|$. The fact that the number of 1 dimensional spaces in $\mathbb{F}^{d}$ can be at most $\frac{q^{d}-1}{q-1}$ completes the proof. Note that the rank of $M_{f}$ can be over any field (we choose $\mathbb{R}$ ).

We get an immediate corollary. Any function $f$, such that the adjacency matrix of $M_{f}$ of the bipartite graph $G_{f}$ is of full rank $2^{n}$ over some field must have upd $(f)=\Omega(n)$. There are several Boolean functions with this property, well-studied in the context of communication complexity (see textbook [8]). Hence, we have for $f \in\left\{\mathrm{IP}_{n}, \mathrm{EQ}_{n}, \mathrm{INEQ}_{n}, \mathrm{DISJ}_{n}, \mathrm{PAL}_{n}^{q}\right\}$, $\operatorname{upd}_{\mathbb{F}}(f)$ is $\Omega(n)$ for any finite field $\mathbb{F}$.

For arguing about $\mathrm{PAL}_{n}^{q}$, it can be observed that the graph is strongly regular (as $q \equiv 1$ mod 4) and hence the adjacency matrix has full rank over $\mathbb{R}[2]$. Except for $\operatorname{PAL}_{n}^{q}$, all the above functions have $O(n)$ sized deterministic branching programs computing them and hence the Pudlák-Rödl theorem (Theorem 2.1) gives that upd for these functions (except $\left.\mathrm{PAL}_{n}^{q}\right)$ are $O(n)$ and hence the above lower bound is indeed tight.

From Lemma 4.3, it follows that the function $\mathrm{SI}_{d}$ also has rank $2^{\Omega\left(d^{2}\right)}$. To see this, it suffices to observe that $\mathcal{P}_{d}$ appears as an induced subgraph in the bipartite realization of $\mathrm{SI}_{d}$. Thus, upd $\left(\mathrm{SI}_{d}\right)$ is $\Omega\left(d^{2}\right)$. We proved in Theorem 3.3 that $\mathrm{pd}\left(\mathrm{SI}_{d}\right)=d$. This establishes a quadratic gap between the two parameters. This completes the proof of Theorem 1.1.

Let $D(f)$ denote the deterministic communication complexity of the Boolean function $f$. We observe that the rectangle argument used in the proof of Lemma 4.5 is similar to the matrix rank based lower bound arguments for communication complexity. This yields the Proposition 4.6. If upd $(f) \leq D$, the assignment also gives a partitioning of the 1 s in $M_{f}$ into at most $\frac{q^{D}-1}{q-1} 1$-rectangles. However, it is unclear whether this immediately gives a similar partition of 0 s into 0 -rectangles as well. Notice that if $D(f) \leq d$, there are at most $2^{d}$ monochromatic rectangles (counting both 0-rectangles and 1-rectangles) that cover the entire matrix. However, our proof does not exploit this difference.

- Proposition 4.6. For a Boolean function $f:\{0,1\}^{n} \times\{0,1\}^{n} \rightarrow\{0,1\}$ and a finite field $\mathbb{F}, \operatorname{upd}_{\mathbb{F}}(f) \leq 2^{D(f)}$ and $D(f) \leq\left(\operatorname{pd}_{\mathbb{F}}(f)\right)^{2} \log |\mathbb{F}|$

Proof. We give a proof of the first inequality. Any deterministic communication protocol computing $f$ of cost $D(f)$, partitions $M_{f}$ into $k$ rectangles where $k \leq 2^{D(f)}$ rectangles. Define $f_{i}:\{0,1\}^{n} \times\{0,1\}^{n} \rightarrow\{0,1\}$ for each rectangle $R_{i} i \in[k]$, such that $f_{i}(x, y)=1$ iff $(x, y) \in R_{i}$. Note that $\operatorname{upd}_{\mathbb{F}}\left(f_{i}\right)=1$ and $f=\vee_{i=1}^{k} f_{i}$. For any $(x, y) \in\{0,1\}^{n} \times\{0,1\}^{n}$ if $f(x, y)=1$, there is exactly one $i \in[k]$ where $f_{i}(x, y)=1$. Hence for each $j \in[k], j \neq i$, the intersection vector corresponding to the edge $(x, y)$ in the assignment of $f_{j}$ is trivial. Hence the assignment obtained by applying Lemma 3.1 , to $f_{1}, \vee f_{2} \vee \ldots f_{k}$ will have the property that for any $(x, y)$ with $f(x, y)=1$, the intersection dimension is 1 . Hence $\operatorname{upd}_{\mathbb{F}}(f) \leq k \leq 2^{D(f)}$. To prove the second inequality, consider the protocol where Alice sends the subspace associated with her input as a $\operatorname{pd}_{\mathbb{F}}(f) \times \operatorname{pd}_{\mathbb{F}}(f)$ matrix.

Note that the first inequality is tight, up to constant factors in the exponent. To see this, consider the function $f:\{0,1\}^{n} \times\{0,1\}^{n} \rightarrow\{0,1\}$ whose $\operatorname{pd}_{\mathbb{F}}(f)=\Omega\left(2^{n / 2}\right)[12$, Proposition 
1] and note that $D(f)$ for any $f$ is at most $n$. Tightness of second inequality is witnessed by $\mathrm{SI}_{d}$ since by Lemma $4.3 D\left(\mathrm{SI}_{d}\right)=\Omega\left(d^{2}\right)$ while $\mathrm{pd}\left(\mathrm{SI}_{d}\right)=d$.

\section{Bitwise Decomposable Projective Dimension}

The restriction of intersection dimension being 1, although potentially useful for lower bounds for branching program size, does not capture the branching program size exactly. We start the section by demonstrating a function where the gap is exponential. We show the existence of a Boolean function $f$ such that the size of the optimal branching program computing it is very high but has a very small projective assignment with intersection dimension 1 for any balanced partition of the input.

- Proposition 5.1 (Implicit in Remark 1.30 of [6]). There exist a function $f:\{0,1\}^{n} \times\{0,1\}^{n}$ that requires size $\Omega\left(\frac{2^{n}}{n}\right)$ for any branching program computing $f$ but the upd $(f) \leq O(n)$ for any balanced partitioning of the input into two parts.

The above proposition can be shown by adapting the counting argument presented in Remark 1.30 of [6].

\subsection{A Characterization for Branching Program Size}

Motivated by strong properties observed in Proposition 2.2, we make the following definition.

- Definition 5.2 (Bitwise Decomposable Projective Dimension). Let $f$ be a Boolean function on $2 n$ bits and $G_{f}$ be its bipartite realization. The bipartite graph $G_{f}(X, Y, E)$ is said to have bit projective dimension, $\operatorname{bitp} \operatorname{dim}(G) \leq d$, if there exists a collection of subspaces of $\mathbb{F}_{2}^{d}$ denoted $\mathcal{C}=\left\{U_{i}^{a}\right\}_{i \in[n], a \in\{0,1\}}$ and $\mathcal{D}=\left\{V_{j}^{b}\right\}_{j \in[n], b \in\{0,1\}}$ where a projective assignment $\phi$ is obtained by associating subspace $U_{i}^{a}$ with a bit assignment $x_{i}=a$ and $V_{j}^{b}$ with $y_{j}=b$ satisfying the conditions listed below.

1. for all $(x, y) \in\{0,1\}^{n} \times\{0,1\}^{n}, \phi(x)=\operatorname{span}_{1 \leq i \leq n}\left\{U_{i}^{x_{i}}\right\}, \phi(y)=\operatorname{span}_{1 \leq j \leq n}\left\{V_{j}^{y_{j}}\right\}$ and $f$ is realized by $\phi$.

2. Let $S=\left\{e_{i}-e_{j} \mid i, j \in[d], i \neq j\right\}$. For any $W \in \mathcal{C} \cup \mathcal{D}, \exists S^{\prime} \subseteq S$ such that $W=\operatorname{span}\left\{S^{\prime}\right\}$.

3. for any $S_{1}, S_{2} \subseteq([n] \times\{0,1\})$ such that $S_{1} \cap S_{2}=\phi, \underset{(i, a) \in S_{1}}{\operatorname{span}}\left\{U_{i}^{a}\right\} \cap \underset{(j, b) \in S_{2}}{\operatorname{span}}\left\{U_{j}^{b}\right\}=\{0\}$.

Same property must hold for subspaces in $\mathcal{D}$.

We show that the new parameter bitwise decomposable projective dimension (bitpdim) tightly characterizes the branching program size, up to constants in the exponent.

- Lemma 5.3. Suppose $f:\{0,1\}^{n} \times\{0,1\}^{n} \rightarrow\{0,1\}$ has deterministic branching program of size $s$ then $\operatorname{bitpdim}(f) \leq 6 s$

We show that given a bitpdim assignment for a function $f$, we can construct a branching program computing $f$.

- Theorem 5.4 (Theorem 1.2 restated). For a Boolean function $f:\{0,1\}^{n} \times\{0,1\}^{n} \rightarrow\{0,1\}$ with $\operatorname{bitpdim}(f) \leq d$, there exists a deterministic branching program computing $f$ of size $d^{c}$ for some absolute constant $c$.

Proof. Consider the subspace associated with the variables $\mathcal{C}, \mathcal{D}$ of the bitpdim assignment as the advice string. These can be specified by a list of $n$ basis matrices each of size $d^{2}$. Since $d=\operatorname{bitpdim}(f)=\operatorname{poly}(n)$, the advice string is poly $(n)$ sized and depends only on $n$. 
We construct a deterministic branching program computing $f$ as follows. On input $x, y$, from the basis matrices in $\mathcal{C}, \mathcal{D}$, construct an undirected $\operatorname{graph}^{6} G^{*}$ with all standard basis vectors in $\mathcal{C}, \mathcal{D}$ as vertices and add an edge between two vertices $u, v$ if $e_{u}-e_{v} \in U_{i}^{x_{i}}$ or $e_{u}-e_{v} \in V_{j}^{y_{j}}$ for $i, j \in[n]$. For input $x, y, f(x, y)=1$ iff $G^{*}$ has a cycle. To see this, let $C=C_{1} \cup C_{2}$ be a cycle in $G^{*}$ where $C_{1}$ consists of edges from basis matrices in $\mathcal{C}$ and $C_{2}$ contain edges from basis matrices in $\mathcal{D}$. Note that if one of $C_{1}$ or $C_{2}$ is empty then there is a cycle consisting only of vectors from $\mathcal{C}$ which implies a linear dependence among vectors in $\mathcal{C}$. But this contradicts Property 3 of bitpdim assignment. Hence both $C_{1}$ and $C_{2}$ are non-empty. Then, it must be that $\sum_{(u, v) \in C_{1}} e_{u}-e_{v}+\sum_{(w, z) \in C_{2}} e_{w}-e_{z}=0$. Hence $\sum_{(u, v) \in C_{1}} e_{u}-e_{v}=-\sum_{(w, z) \in C_{2}} e_{w}-e_{z}$. Hence we get a vector in the intersection which gives $f(x, y)=1$. Note that if $f(x, y)=1$, then clearly there is a non-zero intersection vector. If we express this vector in terms of basis, we get a cycle in $G^{*}$.

Hence, to check if $f$ evaluates to 1 , it suffices check if there is a cycle in $G^{*}$ which is solvable in L using Reingold's algorithm [14]. The log-space algorithm can also be converted to an equivalent branching program of size $n^{c}$ for a constant ${ }^{7} c$.

Assuming $\mathrm{C}_{=} \mathrm{L} \nsubseteq \mathbb{\mathrm { L }}$ /poly, the function $\mathrm{SI}_{d}$ (a language which is hard for $\mathrm{C}_{=} \mathrm{L}$ under Turing reductions) cannot be computed by deterministic branching programs of polynomial size. Thus, using Theorem 1.2, we conclude that the function $\mathrm{SI}_{d}$ is a candidate function (under standard complexity theoretic assumptions) for super-polynomial bitpdim lower bounds.

\subsection{Lower Bounds for Bitwise Decomposable Projective dimension}

From the results of the previous section, it follows that size lower bounds for branching programs do imply lower bounds for bitwise decomposable projective dimension as well. As mentioned in the introduction, the lower bounds that Theorem 1.2 can give for bitwise decomposable projective dimension are only known to be sub-linear.

To prove super-linear lower bounds for bitwise decomposable projective dimension, we show that Nechiporuk's method [11] can be adapted to our linear algebraic framework (thus proving Theorem 1.3 from the introduction). The overall idea is the following: given a function $f$ and a bitpdim assignment $\phi$, consider the restriction of $f$ denoted $f_{\rho}$ where $\rho$ fixes all variables except the ones in $T_{i}$ to 0 or 1 where $T_{i}$ is some subset of variables in the left partition. For different restrictions $\rho$, we are guaranteed to get at least $c_{i}(f)$ different functions. We show that for each restriction $\rho$, we can obtain an assignment from $\phi$ realizing $f_{\rho}$. Hence the number of different bitpdim assignments for $\rho$ restricted to $T_{i}$ is at least the number of sub functions of $f$ which is at least $c_{i}(f)$. Let $d_{i}$ be the ambient dimension of the assignment when restricted to $T_{i}$. By using the structure of bitpdim assignment, we count the number of assignments possible and use this relation to get a lower bound on $d_{i}$. Now repeating the argument with disjoint $T_{i}$, and by observing that the subspaces associated with $T_{i}$ s are disjoint, we get a lower bound on $d$ as $d=\sum_{i} d_{i}$.

- Theorem 5.5. For a Boolean function $f:\{0,1\}^{n} \times\{0,1\}^{n} \rightarrow\{0,1\}$ on $2 n$ variables, let $T_{1}, \ldots, T_{m}$ are partition of variables to $m$ blocks of size $r_{i}$ on the first $n$ variables. Let $c_{i}(f)$ be the number of distinct sub functions of $f$ when restricted to $T_{i}$, then $\operatorname{bitpdim}(f) \geq$ $\sum_{i=1}^{m} \frac{\log c_{i}(f)}{\log \left(\log c_{i}(f)\right)}$

6 Note that this is not a deterministic branching program.

7 Using more space efficient methods than [14], the constant $c$ can be estimated to be at most 5 . 
Proof. Let $(x, y)$ denote the $2 n$ input variables of $f$ and $\rho:\left\{x_{1}, \ldots, x_{n}, y_{1}, \ldots, y_{n}\right\} \rightarrow$ $\{0,1, *\}$ be a map that leaves only variables in $T_{i}$ unfixed. Let $\phi$ be a bitpdim assignment realizing $f$ and let $G_{f}(X, Y, Z)$ denote the bipartite realization of $f$. Let $\mathcal{C}=\left\{U_{i}^{a}\right\}_{i \in[n], a \in\{0,1\}}, \mathcal{D}=$ $\left\{V_{j}^{b}\right\}_{j \in[n], b \in\{0,1\}}$ be the associated collection of subspaces. Let $\rho$ be a restriction that does not make $f_{\rho}$ a constant and $(x, y) \in\{0,1\}^{n} \times\{0,1\}^{n}$ which agrees with $\rho$. We use $x, y$ to denote both variables as well as assignment. From now on, we fix an $i$ and a partition $T_{i}$.

Define $L=\operatorname{span}_{i \in[n], \rho(i) \neq *}\left\{U_{i}^{\rho(i)}\right\}$ and $R=\operatorname{span}_{j \in[n]}\left\{V_{j}^{\rho(n+j)}\right\}$. For any $x \in\{0,1\}^{n}$ that agrees with $\rho$ on the first $n$ bits, define $Z^{x}=\operatorname{span}_{j \in T_{i}}\left\{U_{j}^{x_{i}}\right\}$ Note that for any $(x, y)$, which agrees with $\rho$, has $\phi(x)=L+Z^{x}$ and $\phi(y)=R$. For any $f_{\rho_{1}} \not \equiv f_{\rho_{2}}, G_{f_{\rho_{1}}} \neq G_{f_{\rho_{2}}}$. Hence the number of bitpdim assignments is at least the number of different sub functions. We need to give a bitpdim assignment for $G_{f_{\rho}}\left(V_{1}, V_{2}, E\right)$ where $V_{1}=\{x \mid x$ agrees with $\rho\}, V_{2}=\{y\}$ where $y=\rho_{[n+1, \ldots, 2 n]}$ and $E=\left\{(x, y) \mid x \in V_{1}, y \in V_{2}, f(x, y)=1\right\}$. We use the following property to come up with such an assignment.

- Property 5.6. Let $\rho$ be a restriction which does not make the function $f$ constant and which fixes all the variables $y_{1}, \ldots, y_{n}$. For all such $\rho$ and $\forall x, y \in\{0,1\}^{n}$ which agrees with $\rho$, any non-zero $w \in \phi(x) \cap \phi(y)$, where $w=u+v$ with $u \in L$ and $v \in Z^{x}$ must satisfy $v \neq \overrightarrow{0}$.

Proof. Let there exists an intersection vector $w \in\left(L+Z^{x}\right) \cap R$ with $w=u+v, u \in L$ and $v \in Z^{x}$ and $v=\overrightarrow{0}$. Since $\overrightarrow{0} \in Z^{\hat{x}}$ for any $\hat{x}, w=u+\overrightarrow{0}$ is in $L+Z^{\hat{x}}$ and $R$. Thus the function after restriction $\rho$ is a constant. This contradicts the choice of $\rho$.

The assignment $\psi_{\rho}$ for $G_{f_{\rho}}$ defined as: $\psi_{\rho}(x)=Z^{x}$ and $\psi_{\rho}(y)=\underset{x \in V_{1}}{\operatorname{span}}\left\{\Pi_{Z^{x}}\left(R \cap\left(L+Z^{x}\right)\right)\right\}$ Note that for $(x, y) \in V_{1} \times V_{2}, f_{\rho}(x)=f(x, y)$. Following claim shows that $\psi_{\rho}$ realize $f_{\rho}$.

- Claim 5.7. For any $(x, y) \in V_{1} \times V_{2}, f(x, y)=1$ if and only if $\psi_{\rho}(x) \cap \psi_{\rho}(y) \neq\{0\}$.

Proof. For any $(x, y) \in X \times Y, \phi(x) \cap \phi(y) \neq\{0\}$ if and only if $f(x, y)=1$. Since $V_{1} \subseteq X$ and $V_{2} \subseteq Y$, it suffices to prove: $\forall(x, y) \in V_{1} \times V_{2}, \psi_{\rho}(x) \cap \psi_{\rho}(y) \neq\{0\} \Longleftrightarrow \phi(x) \cap \phi(y) \neq\{0\}$.

We first prove that $\psi_{\rho}(x) \cap \psi_{\rho}(y) \neq\{0\}$ implies $\phi(x) \cap \phi(y) \neq\{0\}$. Let $v$ be a non-zero vector in $\psi_{\rho}(x) \cap \psi_{\rho}(y)$. By definition of $\psi_{\rho}(x), v \in Z^{x}$. By definition of $\psi_{\rho}(y)$, there exists a non-empty $J \subseteq V_{1}$ such that $v=\sum_{\hat{x} \in J} v_{\hat{x}}$ where $v_{\hat{x}} \in Z^{\hat{x}}$. Also for every $\hat{x} \in J$, there exists a $u_{\hat{x}} \in L$ such that $w_{\hat{x}}=u_{\hat{x}}+v_{\hat{x}}$ and $w_{\hat{x}} \in R$. Define $u$ to be $\sum_{\hat{x} \in J} u_{\hat{x}}$. Since each $u_{\hat{x}}$ is in $L, u$ is also in $L$. Hence $w=u+v$ is in $L+Z^{x}$. Substituting $u$ with $\sum_{\hat{x} \in J} u_{\hat{x}}$ and $v$ with $\sum_{\hat{x} \in J} v_{\hat{x}}$ we get that $w=\sum_{\hat{x} \in J} u_{\hat{x}}+v_{\hat{x}}=\sum_{\hat{x} \in J} w_{\hat{x}}$. Since each $w_{\hat{x}} \in R, w \in R$. Hence $w \in R \cap\left(L+Z^{x}\right)$ and $w$ is non-zero as $J$ is non-empty.

Now we prove that $\phi(x) \cap \phi(y) \neq\{0\}$ implies $\psi_{\rho}(x) \cap \psi_{\rho}(y) \neq\{0\}$. Let $w$ be non zero vector in $\phi(x) \cap \phi(y)$ with $w=u+v$ where $u \in L$ and $v \in Z^{x}$. By Property 5.6 we have $v \neq \overrightarrow{0}$. By definition $v \in \psi_{\rho}(y)$. Along with $v \in Z^{x}$, we get $\psi_{\rho}(x) \cap \psi_{\rho}(y) \neq\{0\}$.

Let $Z=\underset{j \in T_{i}}{\operatorname{span}}\left\{U_{j}^{0}+U_{j}^{1}\right\}$. We now prove that subspace assignment on the only vertex in the right partition of $G_{\rho}$ which is $\operatorname{span}_{x \in V_{1}}\left\{\Pi_{Z^{x}}(R)\right\}$ is indeed $\Pi_{Z}(R)$.

- Claim 5.8. $\Pi_{Z}(R)=\operatorname{span}_{x \in V_{1}}\left\{\Pi_{Z^{x}}(R)\right\}$.

Proof. We show span $\left\{\Pi_{Z^{x}}(R)\right\} \subseteq \Pi_{Z}(R)$. Note that $\operatorname{span}_{x \in V_{1}}\left\{\Pi_{Z^{x}}(R)\right\}=\operatorname{span}_{x \in V_{1}, w \in R}\left\{\Pi_{Z^{x}}(w)\right\}$. For an arbitrary $x \in V_{1}$ and $w \in R$, let $v=\Pi_{Z^{x}}(w)$. By definition of $Z^{x}$ and the fact that 
$\left\{U_{i}^{b}\right\}_{i \in[n], b \in\{0,1\}}$ are disjoint, $\Pi_{Z^{x}}(w)=+_{i \in[n], \rho(i)=*} \Pi_{U_{i}^{x_{i}}}(w)$. As $Z=\operatorname{span}_{j \in T_{i}}\left\{U_{j}^{0}+U_{j}^{1}\right\}$, every $\Pi_{U_{i}^{x_{i}}}(w) \in \Pi_{Z}(R)$. Hence the span is also in $\Pi_{Z}(R)$.

Now we show that $\Pi_{Z}(R) \subseteq \operatorname{span}_{x \in V_{1}}\left\{\Pi_{Z^{x}}(R)\right\}$. Let $T_{i}=\left\{i_{1}, \ldots, i_{k}\right\}$. For $1 \leq j \leq k$ define $x^{j}$ to be $x+e^{j}$ where $x \in\{0,1\}^{n}$ agrees with $\rho$ and for any index $i \in[n]$ with $\rho(i)=*, x_{i}=0$ and $e^{j} \in\{0,1\}^{n}$ is 0 at every index other than $i_{j}$. Note that for any $j_{1} \neq j_{2}, j_{1}, j_{2} \in T_{i}$, $Z^{x^{j_{1}}} \cap Z^{x^{j_{2}}}=\{0\}$ by Property 3 of Definition 5.2) Also note that $\operatorname{span}_{j \in T_{i}}\left\{Z^{x^{j}}\right\}=\underset{j \in T_{i}}{\operatorname{span}}\left\{U_{j}^{x_{j}}\right\}=$ $Z$. Hence, $\Pi_{Z}(R)=\operatorname{span}_{j \in T_{i}}\left\{\Pi_{Z^{x^{j}}}(R)\right\}$. But $\operatorname{span}_{j \in T_{i}}\left\{\Pi_{Z^{x^{j}}}(R)\right\} \subseteq \operatorname{span}_{x \in V_{1}}\left\{\Pi_{Z^{x}}(R)\right\}$.

For any $\rho$, which fixes all variables outside $T_{i}, Z$ is the same. And since there is only one vertex on the right partition, for different $\rho, \rho^{\prime}, \Pi_{Z}\left(R_{\rho}\right)=\Pi_{Z}\left(R_{\rho^{\prime}}\right)$ implies $\psi_{\rho}=\psi_{\rho^{\prime}}$. Hence to count the number of different $\psi_{\rho}$ 's for different $f_{\rho}$ 's it is enough to count the number of different $\Pi_{Z}(R)$. To do so, we claim the following property on $\Pi_{Z}(R)$.

- Property 5.9. Let $S=\left\{e_{u}-e_{v} \mid e_{u}-e_{v} \in Z\right\}$. Then there exists a subset $S^{\prime}$ of $S$ such that all the vectors in $S^{\prime}$ are linearly independent and $\Pi_{Z}(R)=\operatorname{span}\left\{S^{\prime}\right\}$.

Proof. By the property of the bitpdim assignment, $\forall i \in[n]$ and $\forall b \in\{0,1\}, V_{i}^{b}=\operatorname{span}\left\{F_{i}^{b}\right\}$

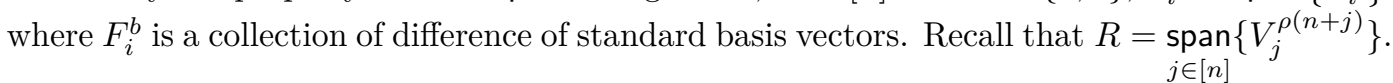
Let $F=\left\{\left(e_{u}-e_{v}\right) \mid e_{u}-e_{v} \in F_{j}^{\rho(n+j)}, j \in[n]\right\}$. Since projections are linear maps and the fact that $F_{j}^{\rho(n+j)}$ spans $V_{j}^{\rho(n+j)}$ we get that, $\Pi_{Z}(R)=\underset{w \in F}{\operatorname{span}}\left\{\Pi_{Z}(w)\right\}$. Since $Z$ is also a span of difference of standard basis vectors, $\Pi_{Z}\left(e_{u}-e_{v}\right)$ is one of $\overrightarrow{0}, e_{u}-e_{w}$ or $e_{w}-e_{v}$ where $e_{w}$ is some standard basis vector in $Z$. Let $S^{\prime \prime}=\cup_{e_{u}-e_{v} \in F} \Pi_{Z}\left(e_{u}-e_{v}\right)$. Hence $S^{\prime \prime} \subseteq S$. Clearly, $\operatorname{span}_{e_{u} \in S^{\prime \prime}}\left\{e_{u}-e_{v}\right\}=\Pi_{Z}(R)$. Choose $S^{\prime}$ as a linear independent subset of $S^{\prime \prime}$.

Property 5.9 along with the fact that $\Pi_{Z}(R)$ is a subspace of $Z$, gives us that the number of different $\Pi_{Z}(R)$ is upper bounded by number of different subsets $S^{\prime}$ of $S$ such that $\left|S^{\prime}\right|=d_{i}$ where $d_{i}=\operatorname{dim}(Z)$. As $S^{\prime}$ is a set of difference of standard basis vectors from $Z,\left|S^{\prime}\right| \leq\left(\begin{array}{c}d_{i} \\ 2\end{array}\right)$. Thus the number of different such $S^{\prime}$ are at most $\sum_{k=0}^{d_{i}}\left(\begin{array}{c}d_{i}^{2} \\ k\end{array}\right)=2^{O\left(d_{i} \log d_{i}\right)}$.

Hence the number of restrictions $\rho$ (that leaves $T_{i}$ unfixed) and leading to different $f_{\rho}$ is at most $2^{O\left(d_{i} \log d_{i}\right)}$. But the number of such restrictions $\rho$ is at least $c_{i}(f)$. Hence $2^{O\left(d_{i} \log d_{i}\right)} \geq c_{i}(f)$ giving $d_{i}=\Omega\left(\frac{\log c_{i}(f)}{\log \left(\log c_{i}(f)\right)}\right)$. Using $d=\sum_{i} d_{i}$ completes the proof.

Theorem 5.5 gives a super linear lower bound for Element Distinctness function. From a manuscript by Beame et.al, ([1], See also [6], Chapter 1), we have $c_{i}\left(E D_{n}\right) \geq 2^{n / 2} / n$. Hence applying this count to Theorem 5.5, we get that $d \geq \Omega\left(\frac{n}{\log n} \cdot \frac{n}{\log n}\right)=\Omega\left(\frac{n^{2}}{(\log n)^{2}}\right)$.

Now we apply this to our context. To get a lower bound using framework described above it is enough to count the number of sub-functions of $\mathrm{SI}_{d}$.

- Lemma 5.10. For any $i \in[d]$, there are $2^{\Omega\left(d^{2}\right)}$ different restrictions $\rho$ of $\mathrm{SI}_{d}$ which fixes all entries other than ith row of the $d \times d$ matrix in the left partition.

Proof. Fix any $i \in[d]$. Let $S$ be a subspace of $\mathbb{F}_{2}^{d}$. Define $\rho_{S}$ to be $\operatorname{SI}_{d}(\mathbf{A}, B)$ where $B$ is a matrix whose rowspace is $S$. And $\mathbf{A}$ is the matrix whose all but $i$ th row is 0 's and $i$ th row consists of variables $\left(x_{i_{1}}, \ldots, x_{i_{n}}\right)$. Thus for any $v \in\{0,1\}^{d}$, rowspace of $\mathbf{A}(x)$ is span $\{v\}$.

We claim that for any $S, S^{\prime} \subseteq S \mathbb{F}_{2}^{d}$ where $S \neq S^{\prime},\left(\mathrm{SI}_{d}\right)_{\rho_{S}} \not \equiv\left(\mathrm{SI}_{d}\right)_{\rho_{S}^{\prime}}$. By definition $\left(\mathrm{SI}_{d}\right)_{\rho_{S}} \equiv \mathrm{SI}_{d}(\mathbf{A}, B)$ and $\left(\mathrm{SI}_{d}\right)_{\rho_{S}^{\prime}} \equiv \mathrm{SI}_{d}\left(\mathbf{A}, B^{\prime}\right)$ where $B$ and $B^{\prime}$ are matrices whose rowspaces 
are $S$ and $S^{\prime}$ respectively. Since $S \neq S^{\prime}$ there is at least one vector $v \in \mathbb{F}_{2}^{d}$ such that it belongs to only one of $S, S^{\prime}$. Without loss of generality let that subspace be $S$. Then $\mathrm{SI}_{d}(\mathbf{A}(v), B)=1$ as $v \in S$ where as $\operatorname{SI}_{d}\left(\mathbf{A}(v), B^{\prime}\right)=0$ as $v \notin S^{\prime}$. Hence the number of different restrictions is at least number of different subspaces of $\mathbb{F}_{2}^{d}$ which is $2^{\Omega\left(d^{2}\right)}$. Hence the proof.

This completes the proof of Theorem 1.3 from the introduction. This implies that for $\mathrm{SI}_{d}$, the branching program size lower bound is $\Omega\left(\frac{d^{2}}{\log d} \times d\right)=\Omega\left(\frac{d^{3}}{\log d}\right)=\Omega\left(\frac{n^{1.5}}{\log n}\right)$ where $n=2 d^{2}$ is the number of input bits of $\mathrm{SI}_{d}$.

\section{Standard Variants of Projective Dimension}

In this section, we study two stringent variants of projective dimension for which exponential lower bounds and exact characterizations can be derived. Although these measure do not correspond to restrictions on branching programs, they illuminate essential nature of the general measure. We define the measures and show their characterizations in terms of well-studied graph theoretic parameters.

- Definition 6.1 (Standard Projective Dimension). A Boolean function $f:\{0,1\}^{n} \times\{0,1\}^{n} \rightarrow$ $\{0,1\}$ with the corresponding bipartite graph $G(U, V, E)$ is said to have standard projective dimension (denoted by $\operatorname{spd}(f)) d$ over field $\mathbb{F}$, if $d$ is the smallest possible dimension for which there exists a vector space $K$ of dimension $d$ over $\mathbb{F}$ with a map $\phi$ assigning subspaces of $K$ to $U \cup V$ such that

- for all $(u, v) \in U \times V, \phi(u) \cap \phi(v) \neq\{0\}$ if and only if $(u, v) \in E$.

- $u \in U \cup V, \phi(u)$ is spanned by a subset of standard basis vectors in $K$.

In addition to the above constraints, if the assignment satisfies the property that for all $(u, v) \in U \times V, \operatorname{dim}(\phi(u) \cap \phi(v)) \leq 1$, we say that the standard projective dimension is with intersection dimension 1 , denoted by uspd $(f)$.

For $N \times N$ bipartite graph $G$ with $m$ edges, consider the assignment of standard basis vectors to each of the edges and for any $u \in U \cup V, \phi(u)$ is the span of the basis vectors assigned to the edges incident on $u$. Moreover, the intersection dimension in this case is 1 . Hence for any $G, \operatorname{spd}(G) \leq \operatorname{uspd}(G) \leq m$. We show that $b c\left(G_{f}\right)=\operatorname{spd}\left(G_{f}\right)$ and $\operatorname{uspd}\left(G_{f}\right)=b p\left(G_{f}\right)$. We refer the reader to the full version [7] for the details of the proof.

Even though $\operatorname{pd}(G) \leq \operatorname{spd}(G)$, there are graphs for which the gap is exponential. For example, consider the bipartite realization $G$ of $\mathrm{EQ}_{n}$ with $N=2^{n}$. We know $\operatorname{pd}(G)=\theta(\log N)$ but $\operatorname{spd}(G) \geq N$ since each of the vertices associated with the matched edges cannot share any basis vector with vertices in other matched edges. Hence dimension must be at least $N$. We show that standard projective dimension of bipartite $G$ is equal to biclique cover number.

\section{Discussion \& Conclusion}

In this paper we studied variants of projective dimension of graphs with improved connection to branching programs. We showed lower bounds for these measures indicating the weakness and of each of the variants.

An immediate question that arises from our work is whether $\Omega\left(d^{2}\right)$ lower bound on $\operatorname{upd}\left(\mathcal{P}_{d}\right)$ is tight. In this direction, since we have established a gap between upd $\left(\mathcal{P}_{d}\right)$ and $\operatorname{pd}\left(\mathcal{P}_{d}\right)$, it is natural to study how pd and upd behave under composition of functions, in order to amplify this gap.

The subspace counting based lower bounds for bitpdim that we proved are tight for functions like $\mathrm{ED}_{n}$. However, observe that under standard complexity theoretic assumptions 
the bitpdim assignment for $\mathcal{P}_{d}$ is not tight. Hence it might be possible to use the specific linear algebraic properties of $\mathcal{P}_{d}$ to improve the bitpdim lower bound we obtained for $\mathcal{P}_{d}$.

Acknowledgements. The authors would like to thank the anonymous reviewers for several suggestions which improved the readability of the paper and specifically for pointing out that the proof of Proposition 5.1 follows from Remark 1.3 in [6].

\section{References}

1 Paul Beame, Nathan Grosshans, Pierre McKenzie, and Luc Segoufin. Nondeterminism and an abstract formulation of Nečiporuk's lower bound method. CoRR, abs/1608.01932, 2016. URL: http://arxiv.org/abs/1608.01932.

2 Béla Bollobás. Random Graphs, Second edition. Cambridge Studies in Advanced Mathematics 73. Cambridge University Press, 2001.

3 Philippe Delsarte. Association schemes and t-designs in regular semilattices. Journal of Combinatorial Theory, Series A, 20(2):230-243, mar 1976. doi:10.1016/0097-3165(76) 90017-0.

4 Péter Frankl and Ronald L. Graham. Intersection theorems for vector spaces. European Journal of Combinatorics, 6(2):183-187, jun 1985. doi:10.1016/s0195-6698(85) 80009-3.

5 Péter Frankl and Richard M. Wilson. The Erdös-Ko-Rado theorem for vector spaces. Journal of Combinatorial Theory Series A, 43(2):228-236, nov 1986. doi:10.1016/ 0097-3165 (86) 90063-4.

6 Stasys Jukna. Boolean Function Complexity: Advances and Frontiers, volume 27 of Series: Algorithms and Combinatorics. Springer New York Inc., 2012.

7 Dinesh Krishnamoorthy, Sajin Koroth, and Jayalal Sarma. Characterization and lower bounds for branching program size using projective dimension. CoRR, abs/1604.07200, 2016. URL: http://arxiv.org/abs/1604.07200.

8 Eyal Kushilevitz and Noam Nisan. Communication Complexity. Cambridge University Press, New York, NY, USA, 1997.

9 Satyanarayana V. Lokam. Complexity lower bounds using linear algebra. Foundations and Trends in Theoretical Computer Science, 4(1\&2):1-155, January 2009. doi:10.1561/ 0400000011.

10 Benjian Lv and Kaishun Wang. The eigenvalues of $q$-Kneser graphs. Discrete Mathematics, 312(6):1144-1147, 2012. doi:10.1016/j.disc.2011.11.042.

11 E. I. Nečiporuk. On a boolean function. Doklady of the Academy of Sciences of the USSR, 164(4):765-766, 1966.

12 P. Pudlák and V. Rödl. A combinatorial approach to complexity. Combinatorica, 12:221226, 1992. doi:10.1007/BF01204724.

13 P. Pudlák and V. Rödl. Some combinatorial-algebraic problems from complexity theory. Discrete Mathematics, 136(1-3):253-279, dec 1994. doi:10.1016/0012-365x (94)00115-y.

14 Omer Reingold. Undirected connectivity in log-space. Journal of the ACM, 55(4):17:117:24, September 2008.

15 Lajos Rónyai, László Babai, and Murali K. Ganapathy. On the number of zero-patterns of a sequence of polynomials. Journal of the AMS, 14:2001, 2002.

16 Heribert Vollmer. Introduction to Circuit Complexity: A Uniform Approach. Springer New York Inc., 1999. 